\section{Raus aus der Komfortzone und rein in die Konfliktarenen!}

\author{
Auf den ersten Blick sieht man bei der Energiewende große Erfolge: \\ Atomausstieg, Paris-Ziele, Kohleausstieg und ein $\mathrm{CO}_{2}$-Preis. \\ Auf den zweiten Blick wird klar: Die Energiewende geht viel zu lang- \\ sam voran, die Politik scheut sich vor vielen Klimaschutzkonflikten, \\ und allzu oft wird nur über Ziele debattiert und nicht gehandelt. \\ Das muss sich ändern. Von Bernd Hirschl
}

$K^{1}$ imaschutzpolitik ist oft anstrengend und komplex. Mit der Energiewende bei Erzeugung und Verbrauch, mit Dezentralisierung, Dekarbonisierung und Energieeinsparvorgaben greifen wir tief in die Lebenswelten von Wirtschaft und Gesellschaft ein. Dies wird durch die sogenannte Sektorenkopplung noch erhöht: der schwankende erneuerbare Strom wird mit allen anderen energierelevanten Sektoren gekoppelt, um diese vollständig $\mathrm{zu}$ dekarbonisieren und gleichzeitig für die Systemstabilität zu nutzen. Es sind also alle Wirtschaftsbereiche, Infrastrukturen und Bürger/innen betroffen. In der Folge treten in allen damit zusammenhängenden Politikfeldern Konflikte auf.

\section{Konflikte ohne Diskurs}

Ein beispielhafter Bereich mit massiven Konflikten ist die Wärmewende im Gebäudesektor. Hier müsste im Grunde stark in das Handeln privater und öffentlicher Immobilienunternehmen, Genossenschaften, privater Eigentümer/innen und Mieter/innen eingegriffen werden also einem sehr relevanten Anteil an Lobbygruppen und Wähler/innen. Kein Wunder, dass die Politik hiervor seit Jahren zurückschreckt und sich lediglich traut, finanzielle Anreize anzubieten und auf stark steigende fossile Energiepreise und technologische Wunder zu hoffen. Eine Hoffnung, die sich bisher nicht erfüllt hat - und die auch der aktuelle weiße Ritter Wasserstoff oder das trojanische Pferd der Technologieneutra- lität nicht werden erfüllen können. Viele Studien beklagen die Umsetzungsdefizite zwar - beschränken sich dann aber dennoch auf rechnerisch hergeleitete Forderungen wie z. B. einen Anstieg der energetischen Sanierungsraten - statt sich intensiv mit der Frage zu beschäftigen, wie wir eine Erhöhung der Sanierungsraten realistisch und sozialverträglich hinbekommen.

Eine solche intensive Auseinandersetzung mit den Umsetzungshemmnissen brauchen wir aber dringend. Nach den gesellschaftlichen Debatten über den Kohleausstieg und einen $\mathrm{CO}_{2}$-Preis muss eine Vielzahl solcher Diskursarenen in allen Konfliktbereichen eröffnet werden, wenn die Energiewende gelingen soll. Und wir brauchen diese - die Komplexität lässt grüßen - auf allen politischen Ebenen, vom Bund bis zu den Kommunen, und in allen Sektoren. So war beispielsweise die Absage einer Kommission zum Thema energetische Gebäudesanierung durch die Bundesregierung ein Fehler - ebenso wie in Berlin einen Mietendeckel einzuführen, ohne die Auswirkungen auf den Klimaschutz zu berücksichtigen. Ein „Klimavorbehalt“, wie ihn sich einige Klimanotstandskommunen oder Landesregierungen vorgenommen haben, kann hier ein hilfreiches Instrument sein. Dabei geht es wohlgemerkt nicht darum, Klilen zu bevorzugen. Es geht darum, konträre Wirkungen aufzuzeigen, um mit parallelen Instrumenten gegenzusteuern - sodass im Idealfall beide Politikmaschutz gegenüber anderen Politikzie- ziele erreicht werden. Aus solchen Debatten können im günstigen Fall sogar beide Politikziele gestärkt hervorgehen und Synergieeffekte gehoben werden.

\section{Beteiligung statt Wegducken}

Für Vermeidungsstrategien und Wegducken bleibt keine Zeit mehr - das stärkt zudem letztlich nur populistische Argumentationsmuster. Wir müssen uns der Frage stellen: Warum kommen Windenergie, Photovoltaik, Geothermie, energetische Gebäudesanierung, Mobilitätswende, Elektromobilität, Bürgerenergie, Flexibilität, Klimaanpassung etc. nicht voran - und was müsste getan werden? Ein ungenügender $\mathrm{CO}_{2}$-Preis ist in allen genannten Bereichen allenfalls ein Teil der Antwort - im Regelfall spielen hier soziale Fragen, Verteilungsgerechtigkeit und ökonomische Teilhabe, ökologische und Flächenkonflikte, Chancen und Risiken digitaler Lösungen, aber auch Bildungs- und Verwaltungsdefizite, Planungsrecht oder rechtliche Komplexität eine gewichtigere Rolle. Diese Themen müssen auf - und die betroffenen Stakeholder und Bürger/innen an einen Tisch. Ob sektorspezifische Kommissionen und begleitende Bürgerräte oder aber runde Tische - wichtiger als das Beteiligungsformat ist die Verpflichtung auf das Ziel der Klimaneutralität, die ja schließlich gesetzlich vorgegeben ist.

Wir müssen aus der Komfortzone von Szenarien und Zielforderungen heraustreten - und uns den sektoralen und politischen Klimaschutzkonflikten in der Umsetzung auf allen Ebenen stellen. Das hat mehr Impact auf das Ziel der Klimaneutralität als die nächste intensive Debatte um schärfere Langfristziele, an die sich letztlich keine Politikerin wirklich binden muss.

\section{AUTOR + KONTAKT}

Dr. Bernd Hirschl ist Leiter des Forschungsfelds "Nachhaltige Energiewirtschaft und Klimaschutz" am IÖW sowie Professor und Leiter des Fachgebiets Management regionaler Energieversorgungssysteme an der BTU Cottbus-Senftenberg. IÖW, Potsdamer Str. 105, 10785 Berlin. E-Mail: Bernd.Hirschl@ioew.de and redistributing the material in any medium or format, provided the original work is properly cited, it is not used for commercial purposes and it is not remixed, transformed or built upon. The access to the digital version of this article is reserved to subscribers of ÖkologischesWirtschaften until two years after the date of publication; after two years it is available to all readers. 